\title{
Interpretation of a complex CME event: Coupling of scales in multiple flux systems
}

\author{
D. Maia ${ }^{1,2}$, G. Aulanier ${ }^{2}$, S. J. Wang ${ }^{3,2}$, M. Pick ${ }^{2}$, J.-M. Malherbe ${ }^{2}$, and J.-P. Delaboudinière ${ }^{4}$ \\ ${ }^{1}$ CICGE, Observatório Astronómico Prof. Manuel de Barros, Faculdade de Ciências da Universidade do Porto, \\ 4430-146 Vila Nova de Gaia, Portugal \\ 2 LESIA UMR CNRS 8100, Observatoire de Paris, 92195 Meudon, France \\ 3 National Astronomical Observatories, Chinese Academy of Sciences, Beijing 100012, PR China \\ 4 Institut d'Astrophysique Spatiale, Université de Paris-Sud-CNRS, Bât. 121, 91405 Orsay, France
}

Received 30 September 2002 / accepted 7 February 2003

\begin{abstract}
Using multi-wavelength observations, in particular in imagery, recorded by SOHO/LASCO-MDI, Yohkoh/SXT, the Meudon spectroheliograph and the Nançay radioheliograph, and performing a linear force-free field extrapolation, we analyzed the triggering and the development of a complex eruptive event in the chromosphere and in the corona. This event included an X1 class flare and an eruptive filament within an active region, but it also involved a whole active complex spanning over 40 degrees of heliolongitude. It resulted in a fast and decelerating partial halo CME, associated with a Moreton wave and a complex series of metric, decimetric and microwave radio bursts. The presence of a coronal null point combined with the occurrence of two distant and nearly simultaneous radio sources give strong arguments in favor of the generalized breakout model for the triggering of the eruption. The observations are consistent with the occurrence of magnetic reconnection at the null point three minutes before the start of the eruption, which is consistent with other observed CME precursors. The analysis of the subsequent development of the event suggests that large interconnecting loops were ejected together with the CME, and that secondary reconnections at low altitude probably occurred remotely in the active complex. Our results show that the triggering and evolution of this complex CME involved multiple magnetic flux systems over a large coronal volume surrounding the flare site, and that it resulted from the coupling of scales from narrow reconnection current sheets to very large inter active region magnetic connections.
\end{abstract}

Key words. Sun: coronal mass ejections (CMEs) - Sun: flares - Sun: radio emission - Sun: magnetic fields

\section{Introduction}

The typical coronal mass ejection (CME) seen by coronagraphs spans about 50 degrees in angular extent, with outstanding events reaching angular extents in excess of 100 degrees (for a review of CME properties see Gosling 1997). Nonetheless, there are many different spatial scales in the corona tied to CME origin, early development, and propagation. The CME phenomenon results from the coupling of scales that go from the very small, like the scales at which reconnection occurs (current sheets), to small active regions and filaments, to very large transequatorial loops and very large coronal dimmings.

The ideal situation would be to combine observations in as many spatial scales as possible. Most CME observations include only the large-scale views given by coronagraphs. Other observations, like those provided by solar disk images, have the possibility to image different spatial scales but given typically very low temporal resolution the observed features become large-scale very fast. Some instruments have relatively good time resolution (a few tens of seconds), but are restricted to a relatively small field of view. Despite these limitations,

Send offprint requests to: D. Maia, e-mail: dmaia@fc.up.pt there were quite recently some important developments that have allowed for a better understanding how the interplay between different spatial scales contributes to CME origin and early development. Many of these works are based on multiinstrument/multi-wavelength observations. Webb et al. (1997) have shown multiple neutral lines involved in CME launch. Large-scale dimmings and "EUV coronal waves" are now well established as being associated with CMEs (Thompson et al. 2000; Delannée et al. 2000), although what the "EUV coronal wave" phenomenon really is remains uncertain (Delannée 2000). Transequatorial loops are an ingredient in some CMEs development (Delannée \& Aulanier 1999; Khan \& Hudson 2000; Pohjolainen et al. 2001). From fast-time-cadence radio imaging observations in the metric domain it is now known that the sites of radio emission tend to follow the latitudinal extent of the coronal mass ejection later seen in coronagraphic images (Maia et al. 1999). The time-scales involved in the magnetic restructuring in the early development of the CME are of the order of a few minutes and may involve magnetic flux systems quite far from the main flaring region (Maia et al. 2001; Delannée et al. 2000; Aulanier et al. 1998; Bagalá et al. 2001; Sterling \& Moore 2001; Sterling et al. 2001). 
From the theoretical and numerical simulation points of view there is also a spatial scale problem. Very complex regions are the nightmare of theoreticians who prefer to model CMEs in simple magnetic geometries. Hence most models of CMEs include small scales only, like active regions (ARs) and filaments. Breakout models (Antiochos et al. 1999) that require the interaction between multiple magnetic flux systems, are intermediate. Rather complex three-dimensional CME models, involving multiple magnetic flux systems (generalized breakout models), are now computationally feasible.

Another problem is how to match theory and modeling efforts with observations. Any comparison must be essentially qualitative, no model nor numerical code has yet progressed to the point of working under fully realistic coronal conditions. At present the best approach seems to be to combine multiwavelength observations with information on how the magnetic field is structured. This can only be done using magnetic field extrapolations to infer the pre-eruptive magnetic topology. This information lets one know where important features like null points, current sheets, separatrix layers and bald patches are situated. This is very important to test which magnetic flux systems participate in the eruption and thus validate some model assumptions.

We present a detailed analysis and discussion, based on multiwavelength observations and magnetic field extrapolations, of the event of October 14, 1999. A CME was observed by the Large-Angle Spectrometric Coronagraph (LASCO; Brueckner et al. 1995) onboard the Solar and Heliospheric Observatory (SOHO), and an impulsive $\mathrm{X} 1 / 1 \mathrm{~N}$ flare is reported in the Solar Geophysical Data. The flare occurred on Active Region 8731, part of a group that spans over 40 degrees in heliolongitude and is structurally quite complex. The sources of data used in this study were the radio images at 5 frequencies from the Nançay Radioheliograph (NRH; Kerdraon \& Delouis 1997, dynamic spectra from a series of radiospectrographs in the frequency band of a few $\mathrm{GHz}$ to a few MHz, Wind/Waves (Bougeret et al. 1995) from 1 to $10 \mathrm{MHz}$, Porto (Magalhães \& Carneiro 1998) from 150 to $650 \mathrm{MHz}$, Ondrejov from 0.8 to $4.5 \mathrm{GHz}$ and Beijing (Fu et al. 1995) from 5.2 to $7.6 \mathrm{GHz}, \mathrm{LASCO} / \mathrm{SOHO}$ white light images, Meudon spectroheliographic movies in $\mathrm{H} \alpha$, EIT/SOHO (Extreme Ultraviolet Imaging Telescope; Delaboudinière et al. 1995) images in HII and MDI/SOHO (Michelson Doppler Imager; Scherrer et al. 1995) photospheric magnetic field information.

\section{Chromospheric and coronal observations}

\subsection{Filament eruption and Moreton wave}

Solar activity was at low levels during the early UT hours of 14 October due to occasional C-class subflares from Region 8731 (see Fig. 1). Activity rose to high levels due to an impulsive $\mathrm{X} 1 / 1 \mathrm{~N}$ flare at 08:58 UT.

The details of the $\mathrm{H} \alpha$ event were studied using the $\mathrm{H} \alpha$ images taken by the Meudon spectroheliograph during this period (with an image approximately every minute). The data include images taken at the center of the line and at $\pm 0.5 \AA$ from the

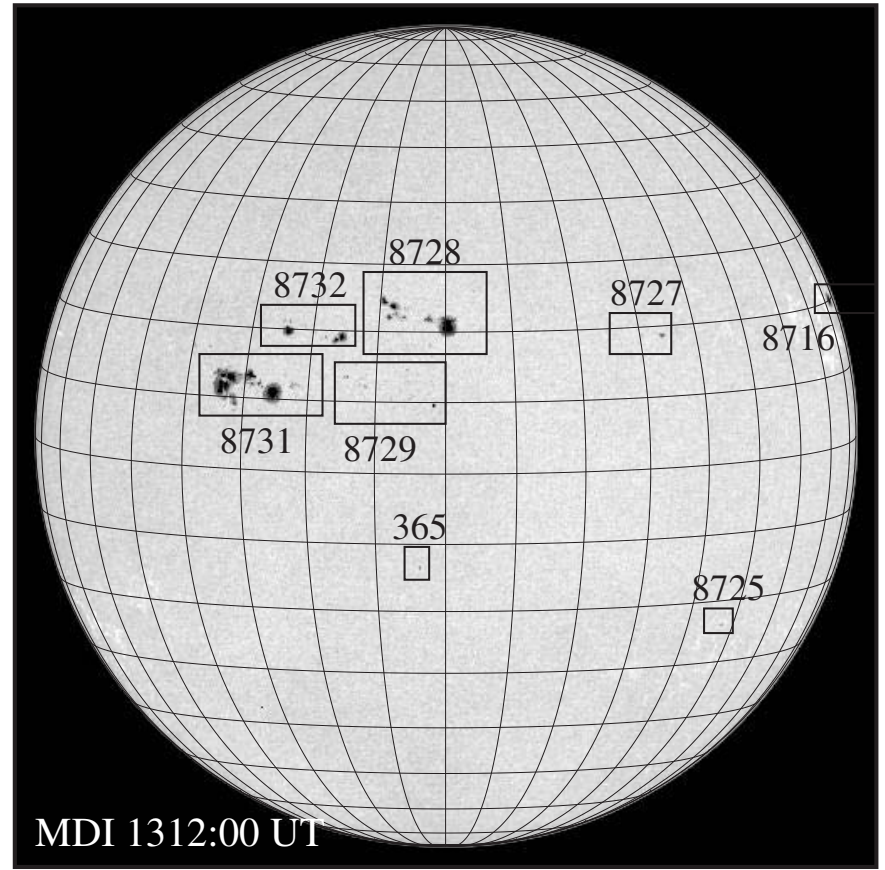

Fig. 1. MDI image showing the position of active regions on October 14, 1999. An impulsive X1/1N flare occured in region 8371 at $0858 \mathrm{UT}$.

center of the line. Important changes are seen in the $\mathrm{H} \alpha$ images taken at 08:58:30 UT when compared to the previous images. Besides a series of bright features seen moving outward (and upward as inferred from the images taken at the wings of the $\mathrm{H} \alpha$ line), a dark narrow feature, encircled by some brighter material, is seen moving also outward (and upward) in the images following 08:59:32 UT. Figure 2 displays the evolution of this feature which progressively approaches the limb. SOHO/EIT was conducting a relatively high-time-cadence program of observations in HeII (304 $\AA$ ), and our outward moving H $\alpha$ feature corresponds quite closely to a filament seen erupting in the HeII images.

A Moreton wave is also identified in the $\mathrm{H} \alpha$ data. Despite being a weak feature, its movement makes it quite easy to detect in movies of the event (in the line and both wings, as expected for a wave). It is also obvious in the running differences shown in Fig. 3. The Moreton wave is first seen after 08:58:30 UT, and its velocity taken at the direction of the filament eruption is (after correcting for projection effects) slightly in excess of $1400 \mathrm{~km} \mathrm{~s}^{-1}$.

\subsection{Development of complex radio activity}

At the onset of the observations, the NRH detected a noise storm in progress at $164 \mathrm{MHz}$ of positive polarization located on the north side of AR 8731. The dominant location of this source is labeled A in Fig. 4 which is a composite of one Halpha image on which the emitting sources prior to the event and the subsequent sources have been reported. A new source seen in position B appeared after 08:34 UT, in coincidence with a small flare occurring in AR 8731. B also has positive polarization, is seen at $164 \mathrm{MHz}$ but barely detected at $236 \mathrm{MHz}$ and 

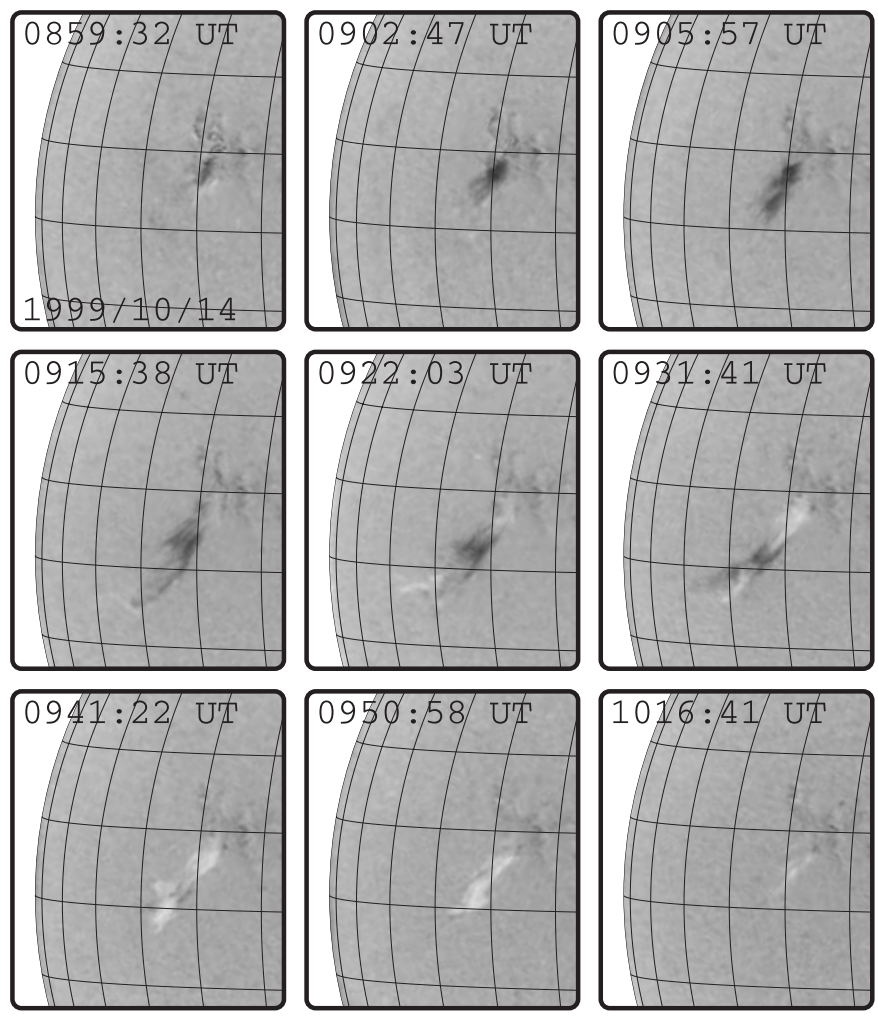

Fig. 2. Series of images built from the $\mathrm{H} \alpha$ information showing the filament eruption. The dark regions correspond to blueshifted (rising) features, while the bright regions correspond to redshifted (falling) features. As we can see the material is rising while moving outward in the first frames, but later on, after 0920 UT we see evidence for inward motion of material falling back.

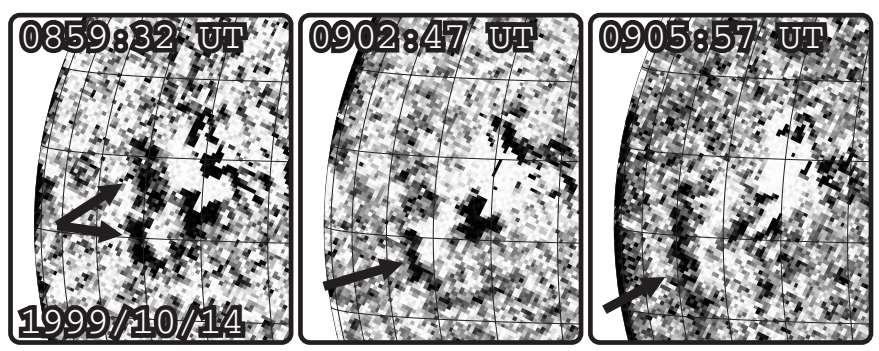

Fig. 3. Running difference images in $\mathrm{H} \alpha$ showing the progression of a Moreton wave indicated by the arrows.

higher frequencies. B region is bursty with three dominant periods around 08:38-08:42 UT, 08:46 UT and 08:52-08:54 UT. At about the same times, some intermittent burst activity of negative polarization started in region $\mathrm{E}$. This activity is an indication of a large loop system.

An intense outburst occurred at about 08:58:10 UT and lasted until 09:02 UT. This outburst was preceded a few seconds before by some weak bursts detected at $236 \mathrm{MHz}$ and/or $164 \mathrm{MHz}$ and was followed by a long-duration continuum. Figure 5 displays the flux evolution of the event measured by the NRH at four frequencies. At $410 \mathrm{MHz}$, the onset of the continuum overlaps with the end of the outburst. A composite spectrum of this event, which was obtained by combining several spectrograms in different frequency ranges, is displayed

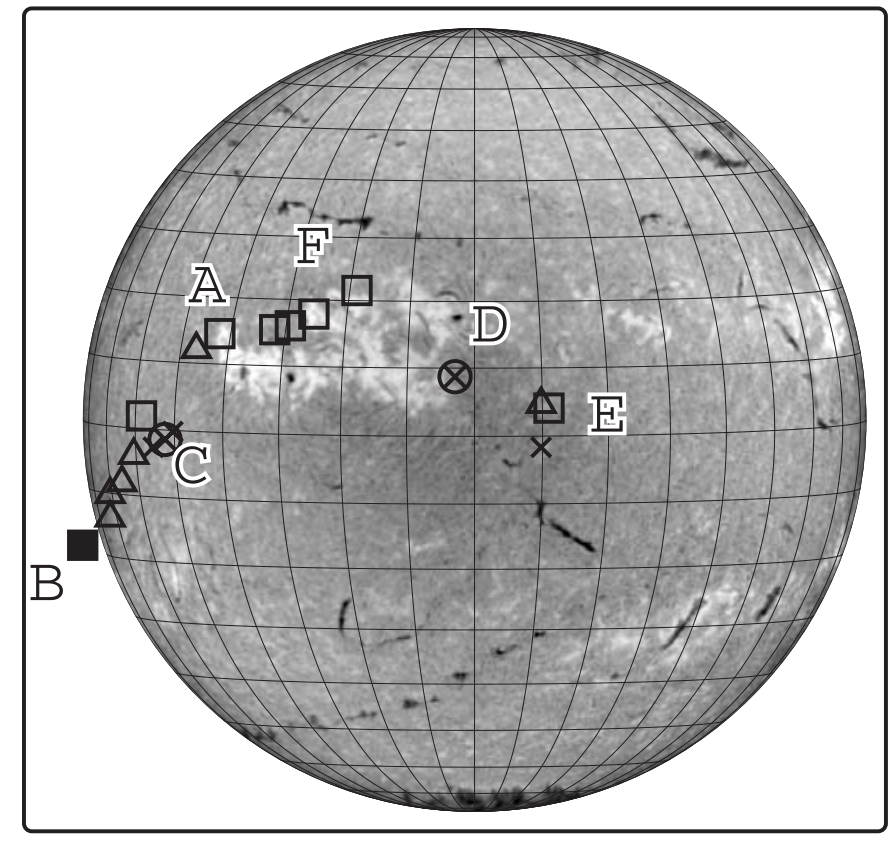

Fig. 4. Position of the main radio emitting regions A, B, C, D, E and F. Different symbols correspond to different observing frequencies: squares to $164 \mathrm{MHz}$, triangles to $236 \mathrm{MHz}$, crosses to $327 \mathrm{MHz}$ and circles to $410 \mathrm{MHz}$.

in Fig. 6. This spectrum shows that the outburst is characterized by series of fast drifting bursts, some of them drifting from 7.6 GHz to below $200 \mathrm{MHz}$. The main emitting source was located in region A. Four other emitting regions labeled $\mathrm{C}$, D, E and F in Fig. 4 contributed to the flux of the outburst. We now describe in more detail their respective temporal and spatial evolution.

Figure 7 shows the flux history of A and D at $410 \mathrm{MHz}$. At this frequency even sources $\mathrm{E}$ and $\mathrm{D}$, which are quite close to each other, are well resolved. A and D are delayed only by a few tens of seconds and are of opposite polarization (A being positively polarized). D source is complex and presents at least two components. The main feature revealed by this figure is the near coincidence of the flux rise onsets of $\mathrm{A}$ and $\mathrm{D}$, which are separated by about $500 \mathrm{Mm}$. These are the only relevant outbursts during the NRH observing period and are very close to the time of the $\mathrm{X}$ flare. The peak brightness temperature in the vicinity of source $D$, prior to and after the event, is about $10^{6} \mathrm{~K}$ (quiet Sun thermal emission). The peak brightness temperature in the vicinity of the A source before and and after the event is about 2 orders of magnitude lower than during the event. It is thus highly unlikely these two outbursts occuring so close in time and being the only major brightness enhancements during a period of 7 hours, are unrelated.

Figure 8 upper panel shows that the emission from source $\mathrm{C}$ is characterized at $236 \mathrm{MHz}$ by a succession of impulsive increases lasting for $10 \mathrm{~s}$. Each increase is itself composed of fast bursts. The positions of these successive pulses drifts southward between 08:58:10 UT and 09:00:30 UT, corresponding to the triangles shown in Fig. 4 in the region labeled $\mathrm{C}$. At $164 \mathrm{MHz}$, one single location is observed. We note that these positions are closely associated in time with the passage 

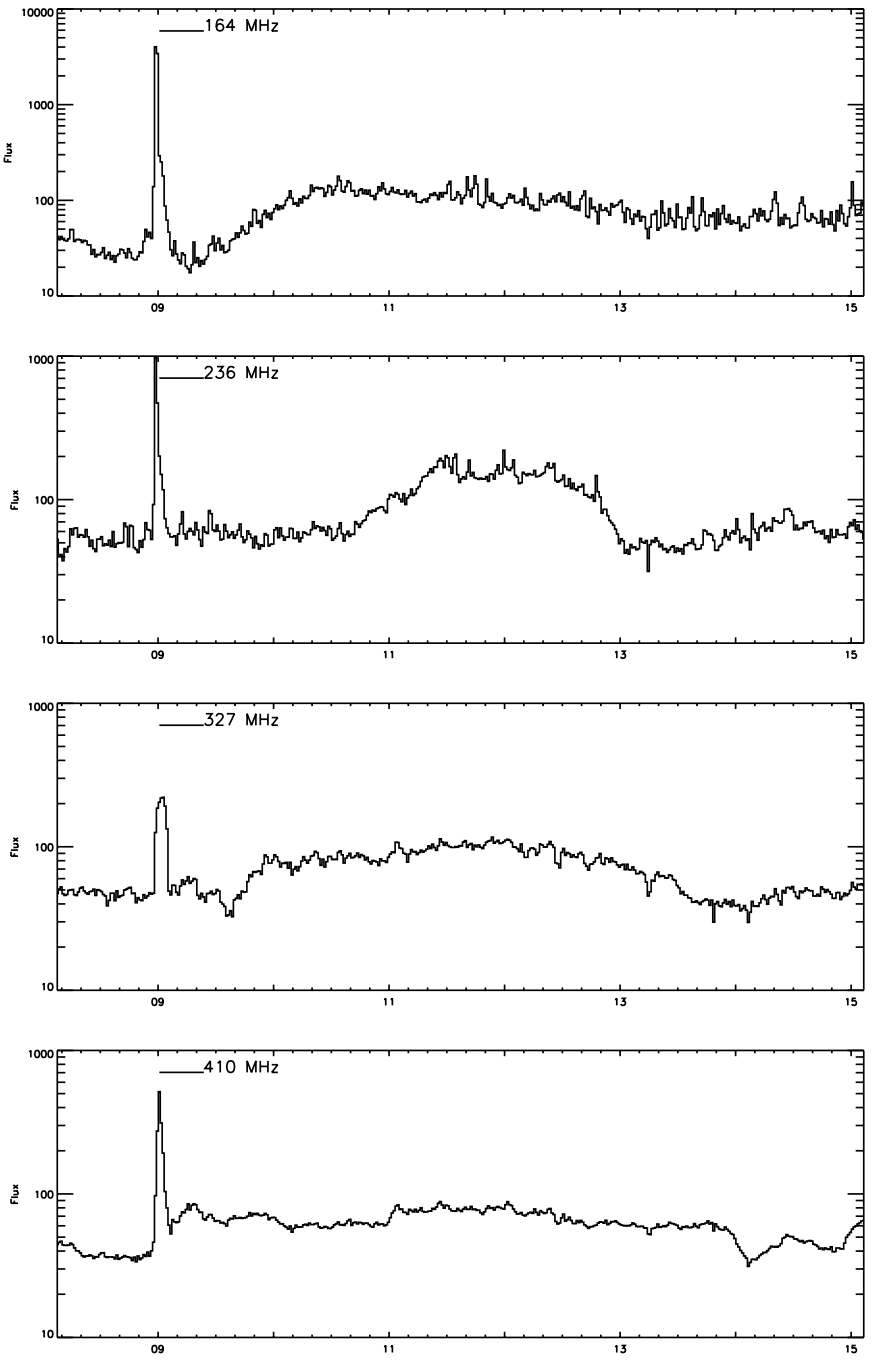

Fig. 5. Flux versus time of the event at the different observing frequencies of the NRH. After a relatively quiet period with only weak bursts at low frequencies, the flux raises by a few orders of magnitude shortly after 08:58 UT. This outburst that lasts a few minutes is then followed by relatively stable or low rising continua at the different frequencies.

of the Moreton wave at this longitude. Appearance of source C was followed about $30 \mathrm{~s}$ later by the occurrence of burst activity in region E. This activity, which is shown in Fig. 8, third panel from the top, was not detected at frequencies below $327 \mathrm{MHz}$. The flux evolution reported in this panel is also composed of a continuum emission coming exclusively from region $\mathrm{D}$, detected at higher frequencies as discussed previously but also at $164 \mathrm{MHz}$ (see Fig. 8, panel 2 from the top). The positions measured at all frequencies coincide.

Note that, due to the appearance at $164 \mathrm{MHz}$ of another emitting region $\mathrm{F}$ having a flux intensity exceeding by more than two orders of amplitude D+E, the flux of these sources cannot be measured properly at $164 \mathrm{MHz}$ after 09:59:30 UT.

To the north of the active regions (8731-8732-8728), a new activity composed of succession of impulsive bursts detected at $164 \mathrm{MHz}$ started suddenly at 08:58:50 UT. Figure 4 shows that the emitting region $\mathrm{F}$ is located westward of the noise storm source A. Flux evolution and position displacement

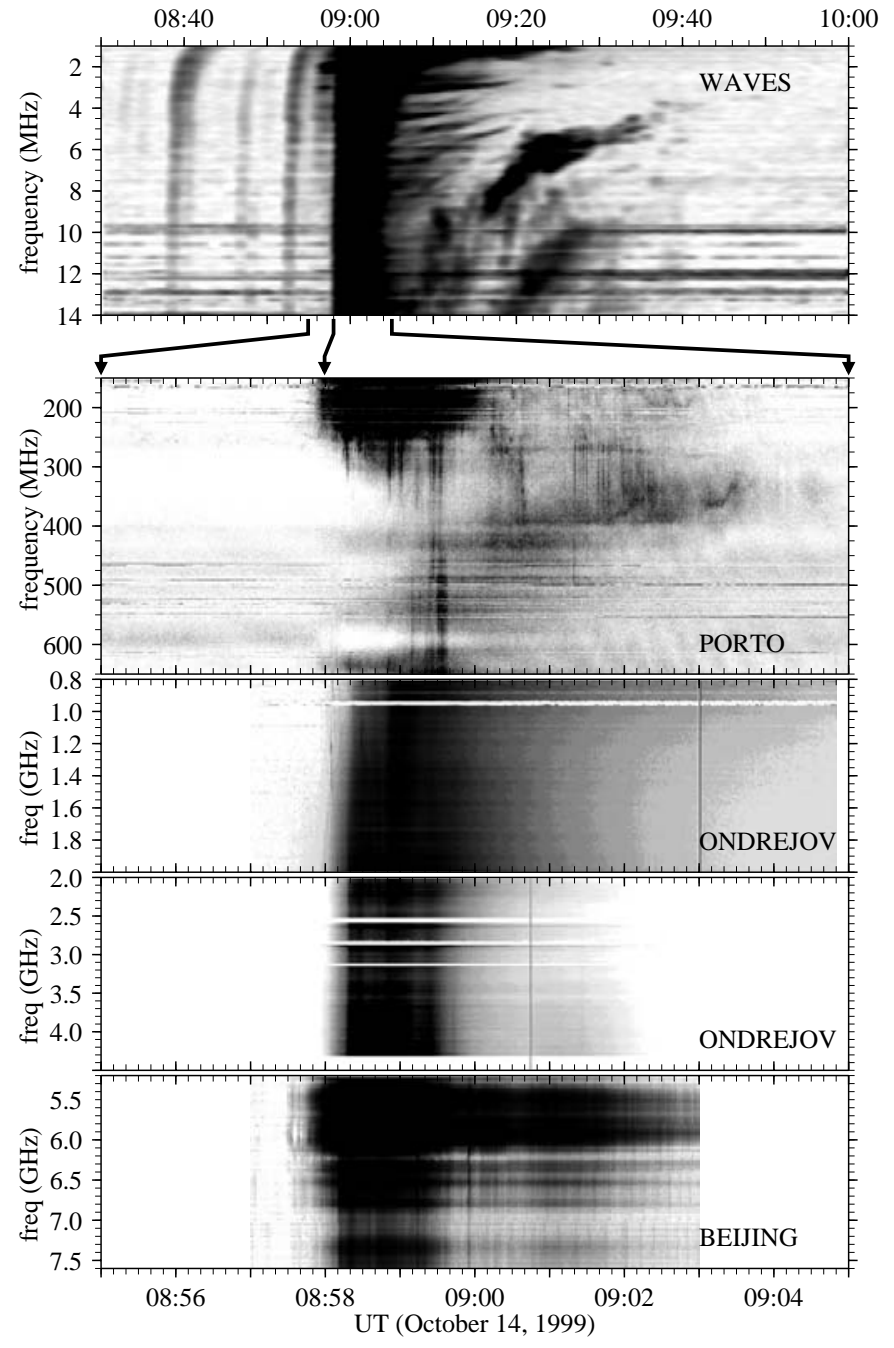

Fig. 6. Composite spectrum in the band of $1 \mathrm{MHz}-7.6 \mathrm{GHz}$, observated by Wind/Waves (1-10 MHz), Porto (150-650 MHz), Ondrejov $(0.8-4.5 \mathrm{GHz})$ and Beijing $(5.2-7.6 \mathrm{GHz})$.

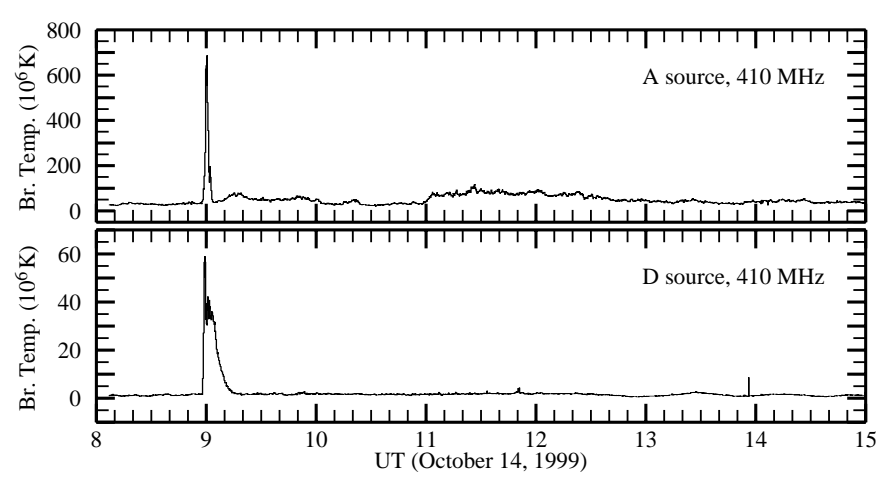

Fig. 7. Peak brightness temperature time at 410 for small boxes encompassing sources A and D. The only relevant outbursts are seen a few seconds after 0858 UT, nearly simultaneous at the two positions (brightness at D starts rising some 20 to $30 \mathrm{~s}$ later than at A).

versus time are shown in Fig. 9. F position shifts toward the west with an apparent velocity of $6000 \mathrm{~km} \mathrm{~s}^{-1}$.

After 08:59:23 UT some changes are seen in the $410 \mathrm{MHz}$ images of the $\mathrm{D}$ source, which becomes extremely elongated in 

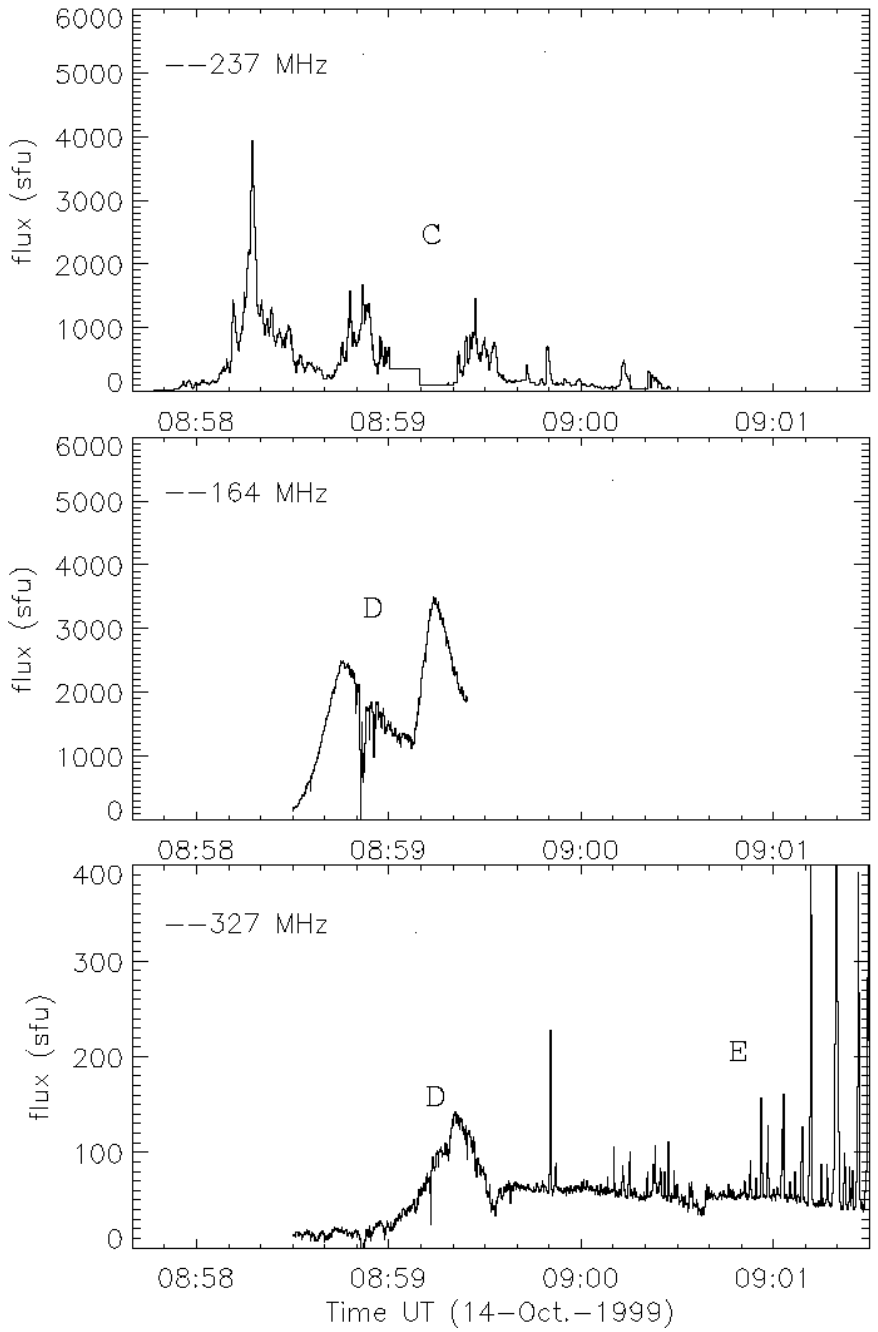

Fig. 8. (From the top) Panel 1: flux evolution of sources $\mathrm{C}$ at $236 \mathrm{MHz}$. Panels 2 and 3: flux evolution of sources $\mathrm{D}$ and $\mathrm{E}$ at $164 \mathrm{MHz}$ and $327 \mathrm{MHz}$. The broad band continuum is associated with D and the bursty emission with $\mathrm{E}$.

the north-west direction. Figure 10 illustrates this by showing brightness profiles taken along the line shown in the radio images in the upper right corner. The profiles before 08:59:13 and after 09:01:33 are roughly Gaussian, and a simple Gaussian fit gives similar widths at half maximum. At 08:59:23 the Gaussian fit gives a slightly broader width; after that the emission shows a skewed profile that cannot be fit by a single Gaussian. At about 09:00:13 a source at about a quarter solar radius westward to the "original" $\mathrm{D}$ source is reasonably well resolved. By subtracting the contribution of a Gaussian component corresponding to the "original" D source we infer that the centroid of the remaining emission moves between 08:59:53 and 09:00:43 with a velocity about $1500 \mathrm{~km} \mathrm{~s}^{-1}$.

In summary, the noticeable features that bring constraints to the interpretation of this complex event are listed as follows: 1. The almost simultaneous onset of activity at two widely separated emission sites A (near the flare site) and D; 2. The excitation of sporadic burst activity in region C, closely associated with the passage of the Moreton wave and followed about $30 \mathrm{~s}$ later by sporadic burst activity of opposite

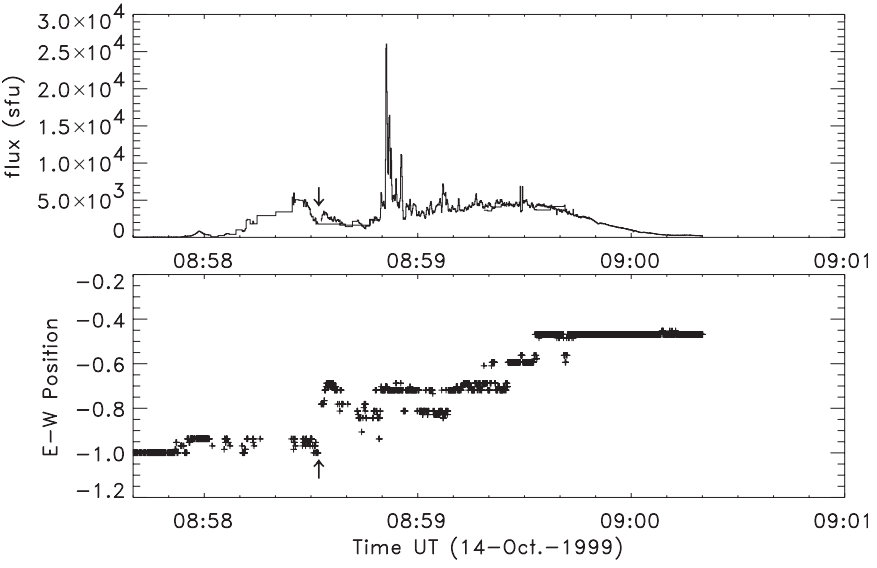

Fig. 9. Flux and location evolution of source F. At about 08:58:30 a new source is seen in radio images at $164 \mathrm{MHz}$. This source shows a displacement toward the north with an apparent speed about $6000 \mathrm{~km} \mathrm{~s}^{-1}$.

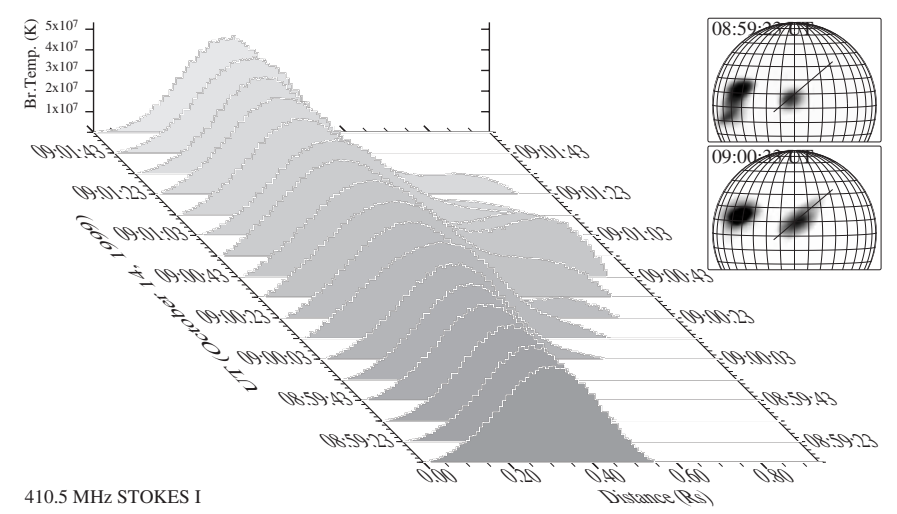

Fig. 10. After 08:59:23 UT the D source becomes extremely elongated in the direction shown by the line in the $410 \mathrm{MHz}$ image in the upper right corner. The brightness profiles taken along this line show an excess brightness that moves with an apparent velocity about $1500 \mathrm{~km} \mathrm{~s}^{-1}$.

polarization in region $\mathrm{E} ; 3$. The occurrence at the north of the of active plage complex of successive bursts showing an apparent displacement of $6000 \mathrm{~km} \mathrm{~s}^{-1} ; 4$. The stretching in the north-west direction of the $\mathrm{D}$ source with an apparent displacement of $1500 \mathrm{~km} \mathrm{~s}^{-1}$.

\section{Dynamics of the coronal mass ejection}

The LASCO observations on October 14, 1999 reveal a coronal mass ejection in close temporal vicinity to the $\mathrm{H} \alpha$ event: the CME is first seen in the C2 field of view at 09:26:05 UT. Figure 11 shows a C2 image taken at 09:26:05 UT. The CME is highly structured in the east limb near the equator, being formed by a series of bright knots, and extends into the southeast quadrant (hardly visible unless one strongly saturates the image). A few features of the CME are identified by arrows in Fig. 11. Feature K1 is in the leading edge of the region of bright knots. Two other features, much brighter than $\mathrm{K} 1$, are labeled K2 and K3; in the 09:26:05 UT image they are clearly behind the leading edge. 

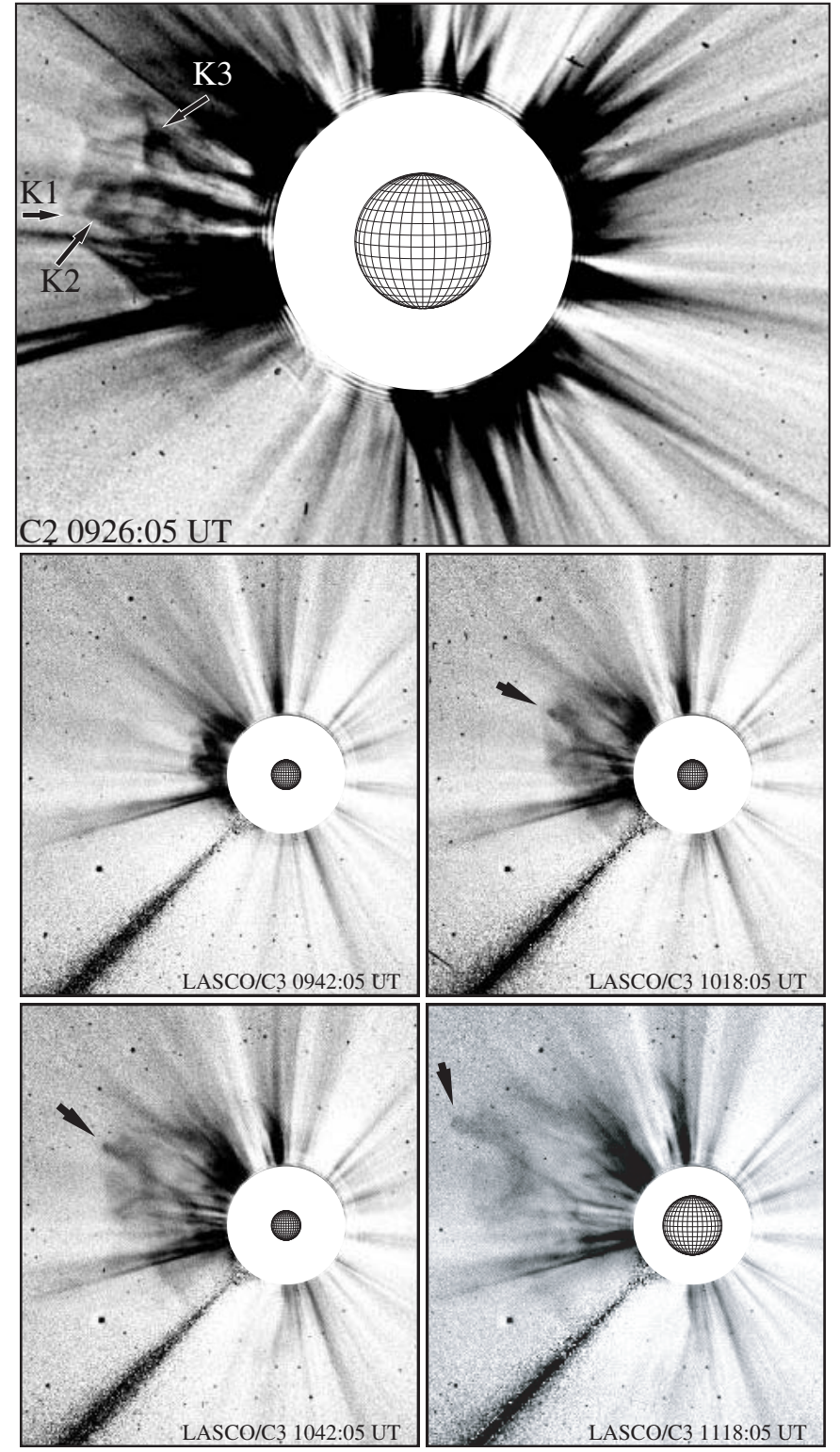

Fig. 11. LASCO images of the CME showing its progression. A series of key figures are indicated in the C2 image at 0926:05 UT. The arrow in $\mathrm{C} 3$ images indicates a feature that seems to correspond to $\mathrm{K} 3$ that expands at a different rate to the rest of the CME.

The CME can then be followed in the C3 field of view and the positions of the several features measured. Figure 12 shows the height-time profile for features K1 and K3. All features have velocity profiles that are very well fit by straight lines (there are no measures below 5 solar radii). Quadratic fits made to the data did not improve significantly the goodness of the fit for any of the features. For none of the features did we find a meaningful acceleration (above 5 solar radii).

The K1 feature moves with a projected velocity of $1000( \pm 20) \mathrm{km} \mathrm{s}^{-1}$. The $\mathrm{K} 2$ feature quite closely follows the K1 feature, with a slightly smaller velocity, consistent with self-similar propagation. Some features taken at the south also show height-time curves consistent with self-similar expansion, as does a feature (seen only in C3) at about 50 degrees north of the equator. The only exception is the bright feature

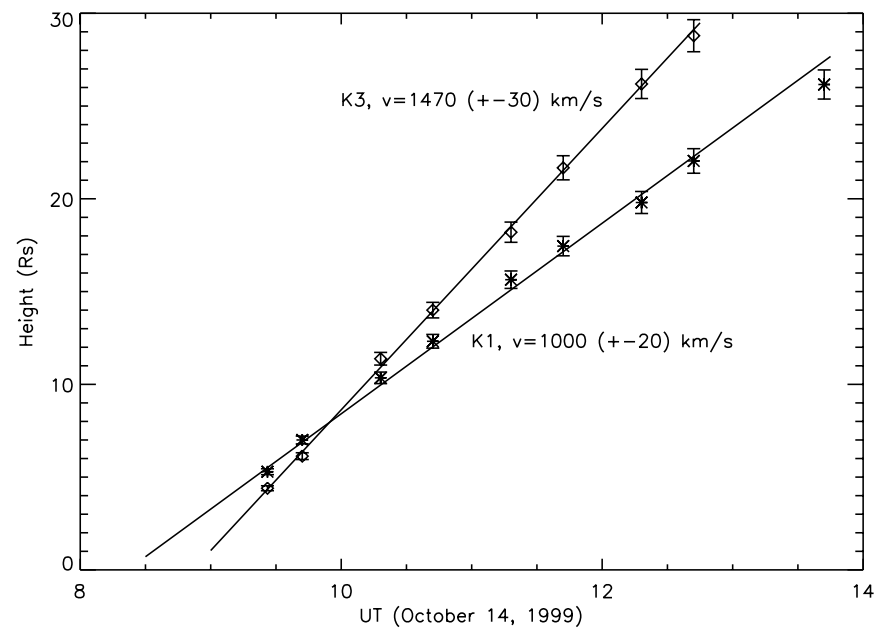

Fig. 12. Height-time plot for features $\mathrm{K} 1$ and $\mathrm{K} 3$ in the CME.

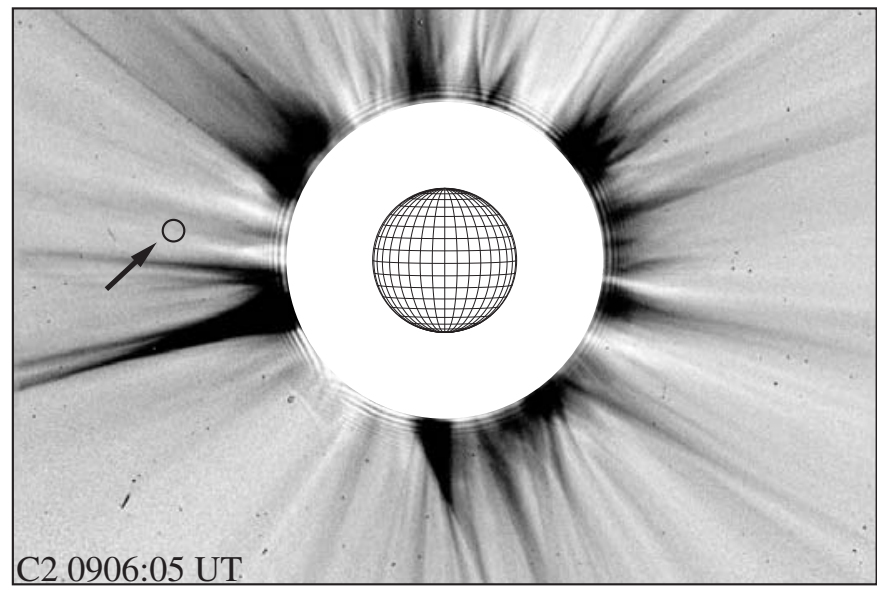

Fig. 13. Decelerated CME? The circle indicated by the arrow shows the extrapolated position of the CME leading edge at this time. No evidence for the CME is seen in the LASCO image.

identified by the arrows in the $\mathrm{C} 3$ images and that seems to correspond quite well to the $\mathrm{K} 3$ feature in $\mathrm{C} 2$. This feature is faster than the other features in the $\mathrm{CME}$, with a projected velocity of $1470( \pm 30) \mathrm{km} \mathrm{s}^{-1}$, and overcomes the other CME features in the C3 image at 10:42:05 UT.

When one looks at previous LASCO images it becomes obvious that the CME height-time curve cannot be linearly extrapolated. LASCO C2 images were available at 08:50 and 09:06 UT. At 09:06 UT one would expect to find the $\mathrm{K} 1$ feature at $3.77 \pm 0.15 \mathrm{Rs}$; the $\mathrm{K} 2$ feature should also be visible in this image. As shown in Fig. 13, no evidence for these features is seen. This means that before 09:26 UT the CME cannot be extrapolated by a linear fit. A constant positive acceleration is also not compatible with the data; the simplest way to explain the absence of the CME is a negative acceleration. To get K1 at 5.3 solar radii in the 09:26 UT image and absent in the 09:06 UT image, this feature would need to travel at an average velocity of $1750 \mathrm{~km} \mathrm{~s}^{-1}$ between the two images. Assuming that the average velocity in the trajectory below the $\mathrm{C} 2$ field of view should be the same or higher, then the CME should take less than $520 \mathrm{~s}$ from 1Rs to reach the 
C2 field of view (about 2.3 solar radii). Hence, the K1 feature could be at 1Rs close to 08:56 UT. Although the absence of coronagraphic data at low altitudes makes all of this somewhat uncertain it is in fact possible (and likely) that the CME is launched in close temporal proximity with the activity seen around 09:00 UT in active region 8731. As described, there is evidence for ejecta from the same region (filament eruption) around 09:00 UT in the $\mathrm{H} \alpha$ and HeII observations.

\section{Interpretation of the triggering of the CME}

We have seen in the previous sections that the spatial and temporal distribution of the radio sources are complex. Also, the timing of their development puts strong constraints on interpretations of all the observations in a consistent way. In this section, we extrapolate the magnetic field in the corona from a photospheric magnetogram. The calculated topology permits us to derive a scenario that can explain the development of the radio sources and that suggests indirectly that the $\mathrm{CME} /$ flare event was caused by a magnetic breakout type instability.

\subsection{Calculation of the coronal magnetic field}

We extrapolate the coronal magnetic field above the active complex in the linear force-free field approximation (i.e. $\boldsymbol{\nabla} \times \boldsymbol{B}=\alpha \boldsymbol{B}$, where $\alpha=$ const.) approximation.

Our code calculates the magnetic field in Cartesian geometry $(x, y, z)$ where $z$ is the altitude above the photosphere, with periodic boundaries in $(x, y)$, using the FFT method (Alissandrakis 1981; Démoulin et al. 1997). For lower boundary conditions, we use a $1100^{\prime \prime} \times 990^{\prime \prime}$ portion of the fulldisk MDI magnetogram, centered on the active complex located at $13 \mathrm{~S} 14 \mathrm{E}$, and inserted inside a large box of $3000^{\prime \prime} \times$ $3000^{\prime \prime}$ where $B z(z=0)=0$, to reduce the boundary effects due to the periodicity (all numbers being given for heliographic coordinates). In the magnetogram region, we set $B z(z=0)=B_{\|}(\mathrm{MDI}) / \cos \psi$ where $\psi$ is the angle between the $z$ axis and the line-of-sight. The magnetic field is calculated with $512 \times 512$ mesh points in $(x, y)$, so the spatial resolution is $\mathrm{d} x=\mathrm{d} y=5.9^{\prime \prime}$.

In order to set the best value for the force-free parameter $\alpha$, we perform several extrapolations with different values of $\alpha$, and we select the one which best matches the coronal loops as observed by Yohkoh/SXT. In this way we find $\alpha=-0.15 \times 10^{-8} \mathrm{~m}^{-1}$. This value is very weak as compared to typically sheared active regions (5-10 times weaker, see Aulanier et al. 1998), sigmoids and intermediate filaments (30-50 times weaker, see Aulanier \& Schmieder 2002). So the field is almost potential on the inter active region scale. The resulting field lines are shown in Fig. 14. The sites of the radio sources are also reported on the same figure. We note that sources $\mathrm{C}$ and $\mathrm{E}$ are located along the large south loop system.

\subsection{A complex configuration with multi-flux systems}

From the results of this extrapolation, we can analyze the topology of the magnetic field, and identify key elements in which magnetic reconnection can occur and thus give rise to particles and wave emissions. Many low altitude nulls and a few high altitude nulls are found.

We find in particular one coronal null point located at $z \simeq 26 \mathrm{Mm}$ in the East part of the active complex, shifted to the West from the flare site by $150^{\prime \prime}$. This null is caused by the large-scale multi-polarity of the magnetic field between AR 8731 (in which the flare occurs) and AR 8729. Three low altitude nulls ( $z \simeq 6,4,5 \mathrm{Mm}$ ) are also present in the West part of the active complex, in the vicinity of the radio source D. These nulls are due to a local inclusion of negative polarities in the leading positive polarity of AR 8731. Figure 15 shows colored magnetic field lines which are very close to the separatrices associated with these four nulls.

\subsection{Indirect evidences of magnetic breakout}

The first observational conclusion derived from the radio observations regarding the triggering of the event is that the impulsive part of the eruptive event (not only the flare, but also the large-scale corona involved in the CME) occurred over $3 \mathrm{~min}$ only, while almost no other activity was detected hours before and after the event. This strongly suggest that all the distant radio active sources were related, and that their occurrence did not result from a "fortunate coincidence". The second conclusion derived from the radio observations is that, during this 3 min duration, some activity occurred quasi-simultaneously (20 s difference) in AR 8731 near the flare site (source A) and in the western part of AR 8729 (source D). Both sources are horizontally separated by $\sim 470^{\prime \prime}$, which would imply a traveling disturbance at $v \geq 17000 \mathrm{~km} \mathrm{~s}^{-1}$, which is far above any value that the Alfvèn speed can take in the corona. Therefore, it is impossible that the flare induced the activity in D. Since it is very likely that the radio sources were related, the only reasonable explanation for this short time delay is that both activities result in the propagation of MHD waves originating from some remote site in the corona located between the flare and the $\mathrm{D}$ site.

We can conjecture that such MD disturbances may find their origin in the coronal null point located between AR 8731 and AR 8729, which is a natural place for the occurrence of magnetic reconnection. Magnetic reconnection is a natural process which produces sudden changes in the magnetic field, which can lead to strong local Lorentz forces that tend to put the field back in a global equilibrium state. This evolution toward a new magnetic equilibrium, following the coronal reconnection, can only be achieved at large scale by the propagation of MHD waves such as shear/torsional Alfvèn waves. From the length of the magnetic field lines originating from the null, assuming a propagation along the field and a constant Alfvèn speed of $1000 \mathrm{~km} \mathrm{~s}^{-1}$, it is possible to estimate the triggering of this coronal reconnection at about $3 \mathrm{~min}$ before the beginning of the flare/CME.

Figure 15 shows that the flare occurred in one of the side lobes of the separatrix associated with the coronal null point. Thus, this configuration differs from the standard magnetic breakout model for CMEs (Antiochos et al. 1999), however it is fully consistent with the yet-unproved generalized 

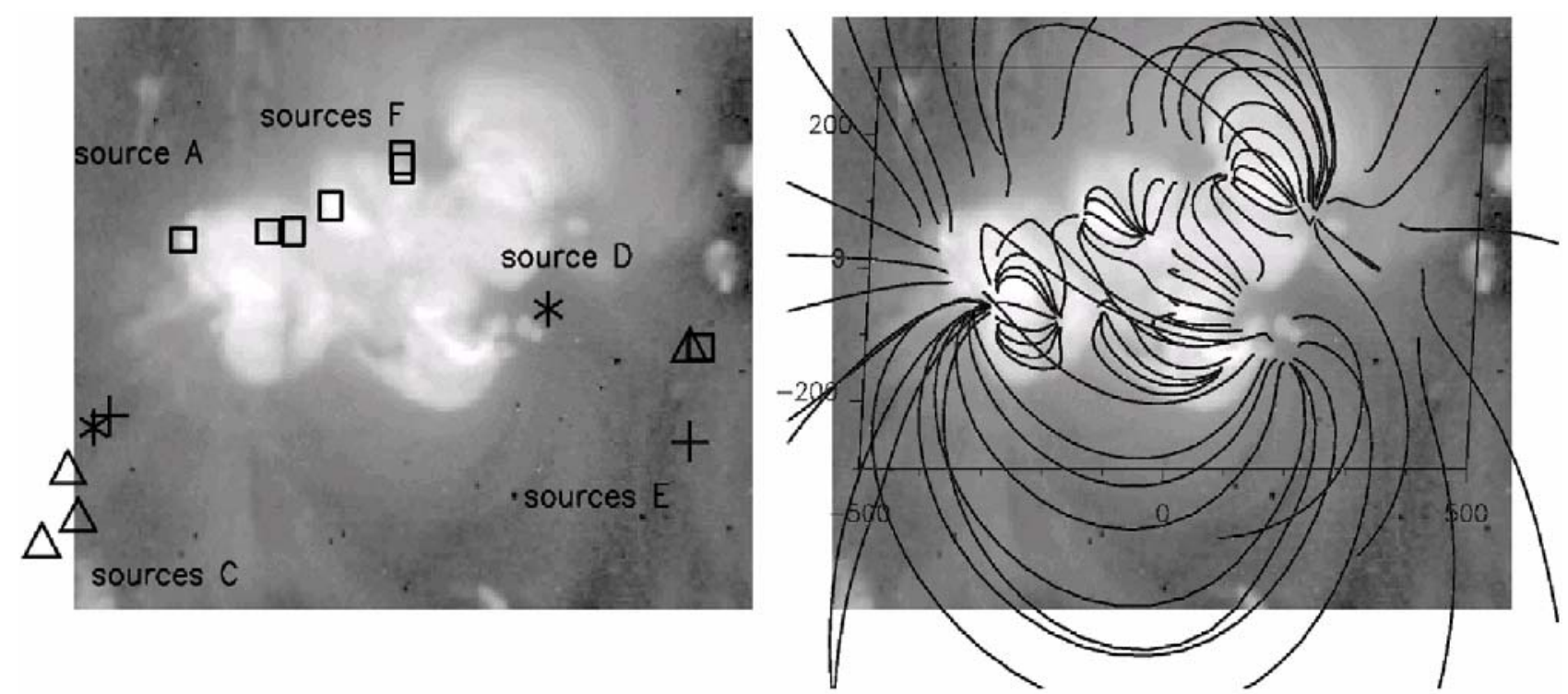

Fig. 14. Left: Yohkoh/SXT image of the active complex (12:42 UT) superimposed with the position of the radio sources observed with the NRH. Right: Same SXT image overlaid by magnetic field lines calculated from the linear force-free field extrapolation of the SOHO/MDI magnetogram (08:00 UT). The thin parallelogram shows a portion only of the magnetogram used for the extrapolation. Units are in arcsec.

magnetic breakout theory proposed by Aulanier et al. (2000) from TRACE observations, also supported by recent EIT observations of "EIT crinkles" (Sterling \& Moore 2001; Sterling et al. 2001).

Unfortunately, due to a lack of high time cadence EIT and TRACE 171 or $195 \AA$ observations, and due to the lack of instruments that can observe at higher radio frequencies than those available with the NRH expected for observing a coronal energy release located at an altitude of $26 \mathrm{Mm}=0.04 R_{\odot}$, we cannot provide any direct evidence for this reconnection. Thus, our interpretation cannot be firmly proved. However the generalized breakout model naturally explains all our available observations in a consistent way, which in our view cannot be done with other existing models except if some observed features are ignored or simply considered to be coincidental.

\section{Interpretation of the further development of the CME}

The F emitting source, which is the strongest and the fastest manifestation of the CME development in the corona, shows an apparent velocity about $6000 \mathrm{~km} \mathrm{~s}^{-1}$. This velocity is far too low to be explained by an electron beam, but also unrealistically high for a coronal shock. Unfortunately no chromospheric or coronal signature is found at other wavelengths, so this prominent feature is not straightforward to interpret. In this section, we present two possible and completely different interpretations, which we further discuss.

\subsection{Coupling of $D$ and $F$ sites through magnetic reconnection}

The first possible interpretation for the emission seen at the F sites is that they are due to electron beams injected in the system of loops that the magnetic field extrapolations calculated as anchored in the vicinity of the region $\mathrm{D}$ and to the North of region A (see the black loops in Fig. 15). We note that the separation of the footpoints of these loops in the vicinity of $\mathrm{D}$ is indeed quite small, and that the $6000 \mathrm{~km} \mathrm{~s}^{-1}$ apparent velocity of source $\mathrm{F}$ would only require a velocity of only $\simeq 1000 \mathrm{~km} \mathrm{~s}^{-1}$ at the footpoints of the loops, which is typically Alfvènic. Radio data shows some evidence for changes in the D source during the period of the F sources: the D source stretches in the northeast direction with a velocity about $1500 \mathrm{~km} \mathrm{~s}^{-1}$. The question is then how to accelerate electrons from these footpoints, which can be answered as follows.

It is noteworthy that the three low-lying magnetic null points located in AR 8729 seem to be well correlated with the radio source $\mathrm{D}$, considering the approximations of a linear force-free field extrapolation. Also, the fact that the radio source D is a composite of two spatially-separated sources (as described in Sect. 2.2) is consistent with the distribution of the nulls: two are very close to each other and one is more shifted to the East). Thus, it is likely that the source D is due to electron beams accelerated from these nulls points during small reconnection events.

The triggering of reconnection in these three low-lying nulls, which are not directly connected by the separatrice of the coronal null, could naturally be provided by the relaxation of the magnetic field lines anchored in the photosphere between them and the footpoint of the spine of the coronal null. This relaxation should then compress the field lines alongside the spine. Subsequent relaxation of the field farther and farther from the footpoint of the spine could then trigger magnetic reconnection in the three null points sequentially, being consistent with the low altitude Alfvènic disturbance as discussed above.

In summary, this first interpretation of the fast moving sources $\mathrm{F}$ is based on the coupling of many different scales. The system of active regions AR 8731 and AR 8729 form a 

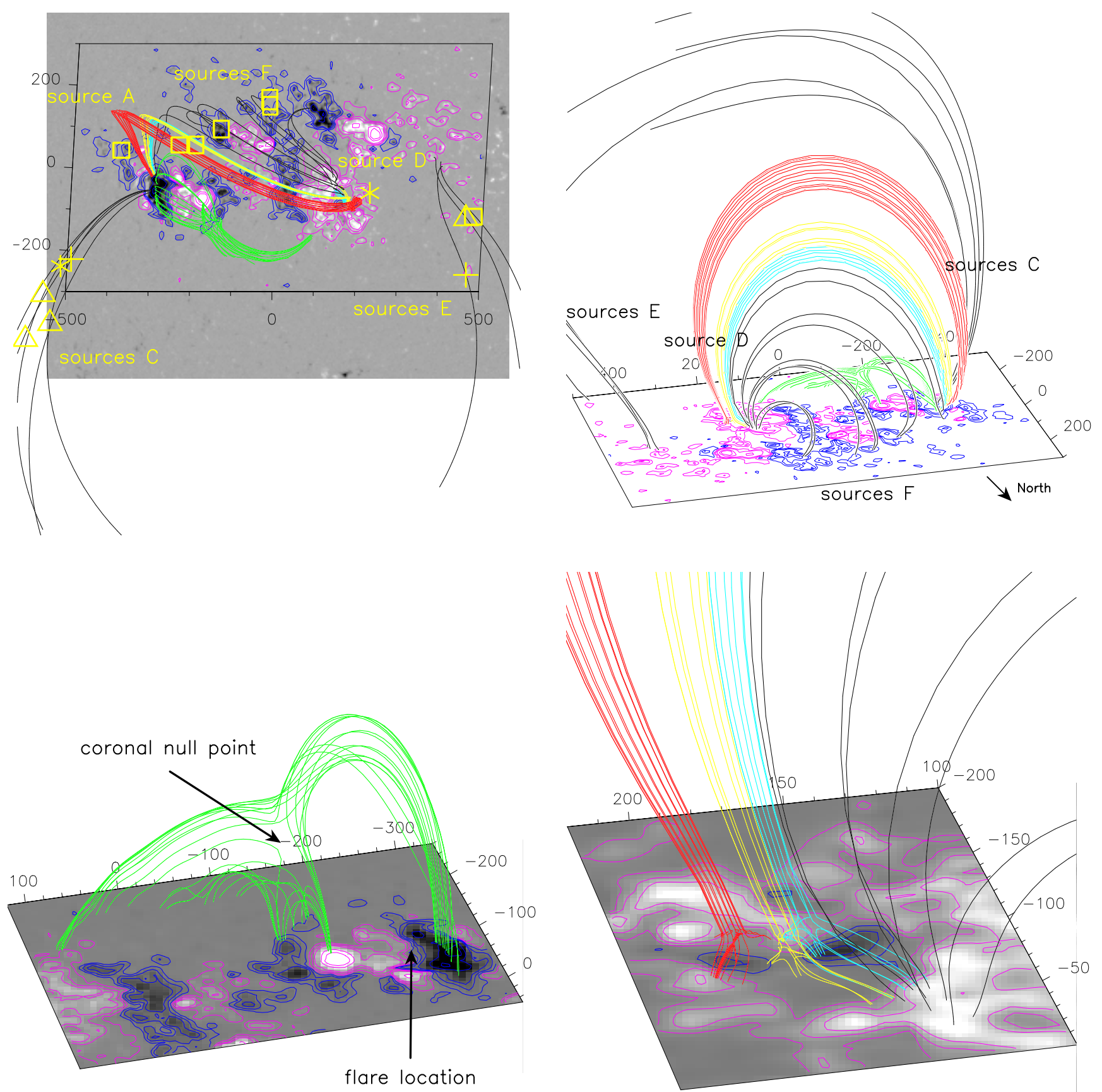

Fig. 15. Top left: SOHO/MDI magnetogram (grey levels) overlaid with the positions of observed radio sources (yellow) and some calculated magnetic field lines. Pink/blue contours stand for $B_{z}(z=0)= \pm 25,75,150,500,800 \mathrm{G}$. The black field lines pass through the radio sources $\mathrm{C}$, E, F. The cyan/yellow/red field lines surround the sepatrices of three nulls points located at low altitude (resp. at $z \simeq 6,4,5 \mathrm{Mm}$ ) in the West part of the active complex. The green field lines surround the separatrix of a coronal null point located in the vicinity of the flare region at $z \simeq 26 \mathrm{Mm}$. Top right: same plot showing the field lines in projection from the North-West. Bottom left: zoom with same projection on the coronal null point, with a vertical stretching factor of 2.5. Bottom right: zoom with same projection on the low altitude null points. Units are in arcsec.

large-scale multi-polar region. This lead to the occurence of a coronal null point above them. Then, during the "breakout reconnection", which we believe is the CME triggering, MHD waves can be emitted from this null point toward the footpoints of its separatrix field lines. When these waves reach these footpoints, they should also be able to excite nearby magnetic structures, in particular the low-lying null points located in AR 8729. Considering the relative locations of these three null points, we conjecture that the magnetic field changes caused by the waves can produce narrow current sheets in the three nulls successively, and thus can drive secondary reconnection events. These reconnections can then accelerate 
electron beams from AR 8729. These electrons can then propagate north-eastward, along large quasi-potential magnetic field lines which interconnect AR 8729 to AR 8732. When they reach $A R$ 8732, they are observed in radio as the $F$ sources, which seem to move very fast due to the successive excitation of the three null points in AR 8729.

\subsection{Shock wave originating from the flare site}

The second interpretation for the fast displacement of the $F$ sources resides in the propagation of a shock, regardless of its blast - or piston driven - nature. This effect has already been reported by Pohjolainen et al. (2001) for another CME event, which is why we mention it here. However we emphasize that in the present event, we found no observational evidence (neither in radio, nor in $\mathrm{H} \alpha$ or in HeII) for a propagating wave/disturbance northward from the flare site.

Although $6000 \mathrm{~km} \mathrm{~s}^{-1}$ is clearly higher than the velocities assumed for coronal shocks, one should note that the data are nonetheless compatible with a coronal shock. It suffices that we consider that this "velocity" is not the shock outward velocity, but rather the result of different shock points intercepting the density layer corresponding to radio emission at $164 \mathrm{MHz}$ at different positions and times.

In fact, the resulting "velocity" can be much higher than the true shock velocity if the shock is quasi-perpendicular to the iso-density levels. For an angle between the shock normal and density gradient of about 15 degrees, the radio sources will show an apparent velocity close to 4 times the shock velocity. We note that the positions of the front of the required hypothetical northern shock are compatible with the onset time and velocity of the Moreton wave detected in the south.

\subsection{Summary}

The first interpretation of the sources $\mathrm{F}$ given above is appealing since it is consistent with all our observations and the calculated magnetic field above the active complex. Also its multi-causal development has several implications that would deserve to be tested in the future. However it is obviously complicated. The oblique shock interpretation is, however, much simpler and more "classical", but it is based on many assumptions regarding the existence of a yet unobserved wave propagating northward, and the coronal density distribution and the directionality of the radio emission, to be consistent with the radio observations.

We favor the first scenario, although we cannot exclude the oblique shock interpretation due to the lack of imaging observations at frequencies below $164 \mathrm{MHz}$. More modeling and new generation radio observations covering many more frequencies than the NRH are clearly needed.

\section{Wide angular extent of the CME}

As is the case for numerous events, the CME studied in this paper covered a large angular fraction of the Sun as observed with SOHO/LASCO (see Fig. 11), whereas it clearly resulted in the eruption of one filament on the scale of a single active region (see Fig. 2).

The on-disk area covered by all the radio sources during the event is consistent with the off-limb angular extent of the $\mathrm{CME}$, after correction for projection effects. On the one hand, this strongly suggests that the whole CME was not only due to the eruptive filament whose dynamic expansion could have covered a large angular extent (this scenario is sketched on the Fig. 22 of Delannée et al. 2000). On the other hand, Figs. 14 and 15 show that the $\mathrm{C}$ and $\mathrm{E}$ radio sources are co-spatial with very large magnetic field lines (calculated from the extrapolation), making long-distance connections over more than 40 degrees between the filament eruption site and a plage region located eastward of the active complex. The occurrence of source E only $30 \mathrm{~s}$ after the rise of the source $\mathrm{C}$ is then fully consistent with the propagation of electron beams along these long loops. We have suggested in Sect. 2.2 that these electrons may be accelerated at $C$ when the Moreton wave encountered these large loops.

The spatial extent of the large southern loops is consistent with the width of the southern part of the CME. Hence, it is very likely that these loops were simply pushed upward from below by an initial transient enveloping the eruptive filament, as described in Delannée \& Aulanier (1999) for a CME related to transequatorial loops. Thus, the development of the radio sources $\mathrm{C}$ and $\mathrm{E}$ do not seem to be related to reconnection occurring in distant originally closed loops, as Maia et al. (1999) and Klein et al. (2001) found in other events.

Note however that the present interpretation can hardly account for the northward extension of the CME, since it would require that the filament also erupted northward, which is neither seen in $\mathrm{H} \alpha$ (see Fig. 2) nor in HeII (EIT images not shown here). Although there is no detected Moreton wave counterpart north of the flare site, the possibility that the radio $\mathrm{F}$ sources seen at $164 \mathrm{MHz}$ may be shock-associated makes possible a scenario similar to the one presented in Pohjolainen et al. (2001).

\section{Summary}

On October 14, 1999 a class X1 flare occurred at 08:58 UT in Active Region 8731. SOHO/MDI magnetograms revealed that this active region was part of a huge multi-polar complex spanning more than 40 degrees in heliolongitude, involving several active regions.

The combined analysis of multi-wavelength observations provided us with the possibility to study extensively many of the chromospheric and coronal manifestations of this complex event that included an $\mathrm{H} \alpha$ Moreton wave propagating toward the South, a small filament erupting toward the South-East, a series of complex radio bursts, showing evidence for electron beams and coronal shock, occurring in the whole active complex, sometimes quasi-simultaneously at long distances from each other, and finally a partial halo CME whose footpoints covered a wide angular extent, which was also decelerated at low altitudes by encountering a previously disturbed coronal medium, which might be due to the passage of an early CME. 
A linear force-free field extrapolation of the MDI magnetogram was performed to calculate the magnetic topology of the active complex prior to the triggering of this chain of events. We found a coronal null point, which defined a fan separatrix enclosing the erupting active region, and a spine field line connecting with the leading part of the active complex. This topology, as combined with the timing and location of the radio bursts provides strong evidence in favor of a generalized breakout scenario for the triggering of the flare/CME (Antiochos et al. 1999; Aulanier et al. 2000) as due to magnetic reconnection at the null point.

We could not directly confirm this model with observations since the altitude of the null point $(z \simeq 26 \mathrm{Mm})$ was too low for its activity to be detectable in radio at the frequencies observable the NRH. However, considering the timing of the radio bursts and the length of the sepatrix field lines, we could estimate a delay of 3 min between the start of the reconnection and the start of the flare/CME. This time delay is consistent with the recent observational evidence of precursors of CMEs observed as jets in EUV (extreme ultraviolet) with TRACE (Aulanier et al. 2000) and in soft X-rays with Yohkoh/SXT (Wang et al. 2002), as well as radio bursts observed in metric wavelengths with the NRH (Pohjolainen et al. 2001).

The subsequent development of radio sources apparently moving super-Alfvènically toward the North could be interpreted as due to secondary reconnections occurring in several low altitude null points at the footpoints of long northward diverging field lines, or by the interaction of a coronal disturbance (maybe associated with the Moreton wave) with the ambient corona. Both scenarios account for the fast displacements of the radio sources, that is then not explained by bulk motions of the sources. As in the trans-equatorial CMEs analyzed in Delannée \& Aulanier (1999) and Wang et al. (2002), the wide angular extent of the CME studied here could be explained by the simple pushing upward of long field lines overlaying the narrow erupting filament and making interconnections between several active regions of the whole complex.

On the modeling side, these interpretations will have to be tested by new MHD calculations. Radio-imaging allows the identification in the corona of non-thermal processes originating from coronal null points. These null points can be located in a variable range of altitudes from the low to the middle corona, as shown in former studies (Wild 1969). New generations of radio-spectro-heliographs such as the Frequency Agile Solar Radiotelescope, FASR (Bastian 2002) should be well suited to observe these null points as CME precursors.

Acknowledgements. Part of this work was supported by the Centre National d'Etudes Spatiales (CNES). D. Maia was partially supported by Fundação para a Ciência e Tecnologia, through the program ESO/FNU/43776/2001 and through grant SFRH/BPD/5521/2001. The visit of S. J. Wang at the Observatoire de Paris was supported through the Sino-Franco cooperation between Observatoire de Paris and National Astronomical Observatories (Beijing) with the financial support of CNRS and CAS, and her work partially supported by the NSFC and MOST grants of China. SOHO is an international collaboration between NASA and ESA. LASCO was constructed by a consortium of institutions: the Naval Research Laboratory (Washington DC, USA), the University of Birmingham (UK), the Max-Planck Institute fur Aeronomie (Katlenburg-Lindau, Germany) and the Laboratoire d'Astronomie Spatiale (Marseille, France). SOHO EIT was built by an international consortium involving ESA and NASA under the supervision of J. P. Delaboudinière. The Nançay Radioheliograph is funded by the French Ministry of Education, the CNRS and the Région Centre.

\section{References}

Alissandrakis, C. E. 1981, A\&A, 100, 197

Antiochos, S. K., Devore, C. R., \& Klimchuk, J. A. 1999, ApJ, 510, 485

Aulanier, G., Schmieder, B., Démoulin, P., van Driel-Gesztelyi, L., \& DeForest, C. 1998, ASPE 97 Meet., Preveza, ASP Conf. Ser., 155, 105

Aulanier, G., DeLuca, E. E., Antiochos, S. K., McMullen, R. A., \& Golub, L. 2000, ApJ, 540, 1126

Aulanier, G., \& Schmieder, B. 2002, A\&A, 386, 1106

Bagalá, L. G., Stenborg, G., Schwenn, R., \& Haerendel, G. 2001, J. Geophys. Res., 106, 225239

Bastian, T. S. 2002, Am. Astron. Soc. Meet., 200

Bougeret, J.-L., Kaiser, M. L., Kellogg, P. J., et al. 1995, Space Sci. Rev., 71, 231

Brueckner, G. E., Howard, R. A., Koomen, M. J., et al. 1995, Sol. Phys., 162, 357

Delaboudiniere, J.-P., Artzner, G. E., Brunaud, J., et al. 1995, Sol. Phys., 162, 291

Delannée, C. 2000, ApJ, 545, 512

Delannée, C., Delaboudinière, J.-P., \& Lamy, P. 2000, A\&A, 355, 725

Delannée, C., \& Aulanier, G. 1999, Sol. Phys., 190, 107

Démoulin, P., Bagalá, L. G., Mandrini, C. H., Hénoux, J. C., \& Rovira, M. G. 1997, A\&A, 325, 305

Fu, Q., Qin, Z., Ji, H., \& Pei, L. 1995, Sol. Phys., 160, 97

Gosling, J. T. 1997, in Coronal Mass Ejections, ed. N. Crooker, J. A. Joselyn, \& J. Feynman (Geophys. Monogr. 99; Washington DC: AGU), 9

Kerdraon, A., \& Delouis, J. 1997, Coronal Physics from Radio and Space Observations, 192

Khan, J. I., \& Hudson, H. S. 2000, Geophys. Res. Lett., 27, 1083

Klein, K. L., Trottet, G., Lantos, P., \& Delaboudinière, J. P. 2001, A\&A, 373, 1073

Magalhães, A., \& Carneiro, J. 1998, Ap\&SS, 261, 211

Maia, D., Vourlidas, A., Pick, M., et al. 1999, J. Geophys. Res., 104, 112507

Maia, D., Pick M., Hawkins, III S. E., Formichev, V. V., \& Jiřička, K. 2001, Sol. Phys., 204, 199

Pohjolainen, S., et al. 2001, ApJ, 556, 421

Scherrer, P. H., Bogart, R. S., Bush, R. I., et al. 1995, Sol. Phys., 162, 129

Sterling, A. C., \& Moore, R. L. 2001, ApJ, 560, 1045

Sterling, A., Moore, R. L., Qiu, J., \& Wang, H. 2001, ApJ, 561, 1116

Thompson, B. J., Cliver, E. W., Nitta, N., Delannée, C., \& Delaboudinière, J. P. 2000, Geophys. Res. Lett., 27, 1431

Wang, T., Yan, Y., Wang, J., Kurokawa, H., \& Shibata, K. 2002, ApJ, 572,580

Webb, D. F., Kahler, S. W., McIntosh, P. S., \& Klimchuck, J. A. 1997, J. Geophys. Res., 102, 224161

Wild, J. P. 1969, Proc. of the Astron. Soc. Australia, 1, 181 